\title{
Graph Based Convolutional Neural Network
}

\section{Michael Edwards}

Xianghua Xie

http://www.csvision.swan.ac.uk
Department of Computer Science

Swansea University

Swansea, UK
In this paper we present a method for the application of Convolutional Neural Network (CNN) operators for use in domains which exhibit irregular spatial geometry by use of the spectral domain of a graph Laplacian, Figure 1. This allows learning of localized features in irregular domains by defining neighborhood relationships as edge weights between vertices in graph $G$. By formulating the domain as a fixed graph representation and projecting the observed data onto $G$ as a graph signal $f$ we are able to utilize the convolution theorem via a graph Fourier transform, matrix multiplication with the columnwise eigenvector matrix $U$, and elementwise multiplication with spectral filters $k$ to learn feature maps (1).

$$
y=U \sum_{i=1}^{I} U^{T} f_{s, i} \odot k_{i, o}
$$

We introduce novel gradient calculations for the convolution operator backpropagation step in regards to both $f(2)$ and $k$ (3). These new calculations are shown to provide higher accuracy and stability compared to calculations presented by [2] and [1].

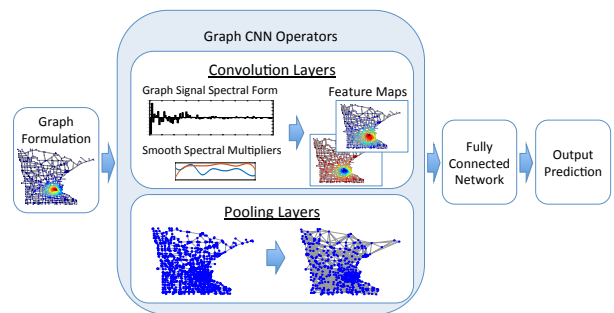

Figure 1: Graph based Convolutional Neural Network components.

The gradient calculation in regards to signal $f_{s, i}$ is given by (2), a spectral convolution of output loss $\nabla y_{s, o}$ with current weights of the spectral filters $k_{i, o}$.

$$
\nabla f_{s, i}=U \sum_{o=1}^{O} U^{T} \nabla y_{s, o} \odot k_{i, o}
$$

Gradients for the spectral filters are provided by (3), which are shown to improve over those of [2] in Figure 2.

$$
\nabla k_{i, o}=\sum_{s=1}^{N} U^{T} \nabla y_{s, o} \odot U^{T} f_{s, i} .
$$

We also present the use of Algebraic Multigrid as a method of graph coarsening, an analogy to the pooling operator of conventional CNNs, agglomerating nodes from the previous layer into a singular node in the subsequent layer. As with standard CNNs this provides both a reduction in graph complexity and generalization of learnt features.

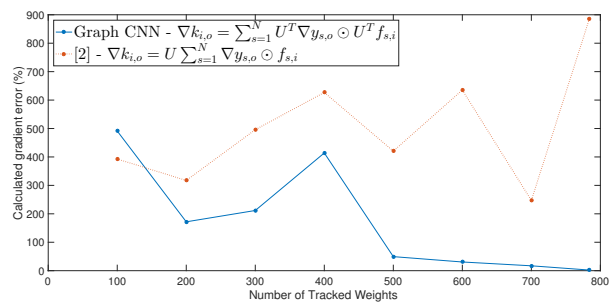

Figure 2: Gradient calculation errors for interpolation of various numbers of tracked weights.

Although this method is adaptable to numerous domains, we evaluate performance on a regular 2D pixel grid and an irregular grid with subsampled spatial geometry with the MNIST digit classification problem projected onto the graph. By utilizing (2) and (3) we obtain accuracy rates of $94.23 \%$ and $94.96 \%$ for the regular and irregular spatial domains respectively.

[1] Joan Bruna, Wojciech Zaremba, Arthur Szlam, and Yann LeCun. Spectral networks and locally connected networks on graphs. CoRR, abs/1312.6203, 2013.

[2] Mikael Henaff, Joan Bruna, and Yann LeCun. Deep convolutional networks on graph-structured data. CoRR, abs/1506.05163, 2015. 